\title{
El Estado Pavoroso de la Locura en la Argentina $^{1}$
}

\author{
[Resumen] \\ En el presente artículo se aborda la problemática de las políticas de reforma psiquiátrica en \\ la actualidad argentina, a partir de un juego presente-pasado con el período alienista. En un \\ segundo momento se reflexiona sobre las condiciones y las líneas de trabajo que estarían \\ presentes en la construcción de un movimiento social por una sociedad sin manicomios. Y \\ en último lugar se analiza cuáles serían los criterios consistentes de justicia para una política \\ en defensa de nuevos servicios públicos para las personas hoy encerradas en el circuito \\ asistencial psiquiátrico. \\ [Palabras claves] \\ Políticas de Salud Mental - Ciudadanía - Desmanicomialización
}

\section{Introducción}

El título precedente no es de mi autoría, pertenece al Dr. Gonzalo Bosch y es parte de un artículo publicado en la Revista «Medicina Argentina» $\mathrm{N}^{\circ} \mathrm{X}$ en 1931. La recuperación del mismo tiene por objeto revisitar la percepción que tenía de la crisis del '30 la psiquiatría institucional, en uno de sus portavoces más representativos. El Dr. Gonzalo Bosch poseía una trayectoria institucional que lo distinguía en su campo: co-fundador de la Liga de Higiene Mental, profesor universitario de Psiquiatría de la Universidad de Bs. As. y de la Universidad del Litoral, autor junto con el Dr. Lanfranco Ciampi de la primera clasificación latinoamericana de las enfermedades mentales y, durante el momento de redacción del texto, interventor en el Hospicio de Mercedes (Borda).

El artículo refleja qué entendía este alienista por «el pavoroso aspecto de la locura en la Argentina», permitiéndonos mapear no sólo la agenda de

\footnotetext{
${ }^{1}$ Este ejercicio que se propone para el artículo tiene afinidades electivas con el juego propuesto por Woody Allen en el film La Rosa Púrpura del Cairo, es decir, la idea de hacer cine dentro del cine. Para el caso que nos convoca, la idea es debatir el tema de la defensa de un grupo social discriminado a partir de la proposición de «una» agenda, en este caso nos planteamos cómo instalar el tema de la asistencia a los locos en un lugar de jerarquía en la agenda social y qué entender por construcción de lo público en el caso de la administración de la locura.
} 
cuestiones de la psiquiatría contemporánea en el período, sino también una serie de diagnósticos y pronósticos que nos sitúan dentro de la invención de un programa de reformas con mayores responsabilidades. Responsabilidades que se traducían en cómo definía el alienismo (con fuerte parecido de familia con los higienistas en el terreno sanitario, los normalistas en el campo educativo, etc.) su participación en la construcción de lo público en la agenda social respecto del caso puntual de cómo administrar el problema social de la locura.

«Crisis» se constituye en palabra de orden de la década del ' 30 , y de hecho atraviesa el discurso del Dr. Gonzalo Bosch en el que conviven fuertes críticas a la deserción del Estado en la materia. Pero la crisis se abate no sólo con un Estado que fracasa, sino que la crisis de legitimación se traslada a la propia psiquiatría y a sus deudas en la resolución del problema de la locura en la Argentina. Dejemos, por lo tanto, que él mismo nos describa los hechos en su lenguaje oficial:

«La República Argentina, que ha conquistado un puesto sobresaliente, en lo que se refiere a organización hospitalaria, digna de las naciones más cultas, se encuentra abocada a problemas de asistencia social de la más grande importancia (...) Para quienes seguimos de cerca los problemas sanitarios y sociales, sabemos que a esta noble lucha en contra de la tuberculosis, de la lepra, del paludismo, debe agregarse la campaña en contra de la locura: otro flagelo, que va pavorosamente aumentando año tras año como en todo país civilizado...Frente a estos hechos se nos ocurre preguntar ¿qué hizo la República Argentina para detener el incremento de la Locura? ¿Organizó un número suficiente de establecimientos en donde todos los enfermos mentales del país puedan recibir un tratamiento adecuado? ¿Las providencias para la asistencia de alienados, responde a criterios científicamente aconsejados por la psiquiatría? Tenemos que responder categóricamente que no. No obstante el hecho de que varios distinguidos colegas (recuerdo aquí solamente a Borda, Ciampi, Ameghino, Obarrio, Gorriti, Mó) hayan levantado su voz de alarma y tratado diversas cuestiones relacionadas con la asistencia del enfermo mental en la Argentina...» (Bosch, 1931:29).

La interrupción del discurso se debe a la importancia de destacar la doble inscripción del Dr. Bosch como funcionario y como representante de las necesidades de los alienados, marca que se repetía en cada uno de los alienistas. A su vez, dos palabras merecen ser contextualizadas: asistencia social y providencia. Las mismas pertenecen a categorías básicas del debate sobre los alcances del Estado en materia social del momento, subrayando la acción social no tanto como derecho de los asistidos, sino como obligación del Estado, responsabilidad que se transfiere al Estado por razones fundamentadas en su propia defensa y en nombre de la seguridad pública.

\section{Un movimiento de reformas, para los funcionarios del disenso}

La historia nos obliga, primero a ser humildes y, segundo, a repetirla o no en la diferencia. Queremos decir con esto, que esta disgresión un tanto larga nos permite disentir con cualquier espíritu fundacional, como primer movimiento social de defensa de políticas «prioritarias» para el caso de la locura en nuestro 
país (de hecho ya a principios de siglo había instituciones estatales y organizaciones no gubernamentales, como la Liga de Higiene Mental, que abogaban por la participación de la salud mental en el ciclo de las políticas públicas y reclamaban su jerarquización, ya había actores preocupados por la locura en la Argentina).

Y la historia también nos permite instalar la diferencia, y no estamos promoviendo la psiquiatrización de la asistencia, sino justamente su «despsiquiatrización», aquí también podemos apelar a las estadísticas y multiplicar los diagnósticos, pero no para re-crear el orden psiquiátrico en la Argentina, aquí también reclamamos el papel del Estado pero para asegurar el derecho a la asistencia, aquí también nos soportamos en las recomendaciones internacionales de la Organización Mundial de la Salud, pero no para otorgar más poder a la psiquiatría aggiornada sino para asegurar, a la atención y no a la exclusión en tanto funcionarios del disenso.

Pero si nos encontráramos en el terreno de definir una visión, por ejemplo, «Por una sociedad sin manicomios», alternativa al orden psiquiátrico, deberíamos recuperar de nuestros mayores «alienistas» la capacidad que demostraron en el terreno de la representación, de la acción colectiva y la producción de autoridad institucional para legitimar el desarrollo de los manicomios en Argentina.

Esta tensión producida nos obliga a reflexionar acerca de qué líneas y con qué agenda de gobierno es posible embarcarse si nos ubicamos a la izquierda, como pretendemos ubicarnos, en el campo de las reformas en salud mental. La contestación a esta pregunta es efectivamente plural, sin embargo, si mi construcción distópica tiene asidero en la realidad, más que incluirnos dentro de un consenso aséptico, creo que debemos partir también del registro de lo antimanicomial para la búsqueda del disenso perdido ${ }^{2}$, justamente para ampliar el discurso de lo posible y promover un nuevo imaginario de reformas, ausente contemporáneamente si somos rigurosos en el análisis.

En los ambientes de la izquierda democrática, ante la crisis de proyectos, constantemente se habla de la invención de nuevas prácticas políticas con el objeto de desmercantilizar y desburocratizar el campo de lo público para promover su efectiva democratización. Creo que nuestra construcción activa de una agenda pública en salud mental también pasa por pensar otras formas de hacer políticas de reformas, diferentes del despotismo esclarecido de la tecnocracia, del inmovilismo a la espera de reformas estructurales y/o de los corporativismos profesionalistas.

Rehabilitar las reformas apunta a la producción de un necesario acortamiento de las distancias entre el movimiento antimanicomial y lo cotidiano de las instituciones, una reforma que apunte fundamentalmente a un movimiento

\footnotetext{
${ }^{2}$ La utopía del disenso viene siendo movilizada por una serie de intelectuales de izquierda, con alto pluralismo en la proposición de las marcas principales que debe adoptar este disenso. Entre los más reconocidos podemos encontrar a P. Anderson, C. Lefort, J. Ranciere y E. Laclau y C. Mouffe, quienes se interrogan principalmente sobre la necesidad de generar una nueva utopía que venga a ocupar el lugar que la utopía proletaria ocupó durante este «corto» siglo XX. En el «Desacuerdo» Jacques Ranciere enuncia la lógica del disenso en el campo de la democracia: «La democracia no es un régimen o un modo de vida social. Es la institución de la política misma, el sistema de las formas de subjetivación por las cuales resulta cuestionado, devuelto a su contingencia, todo orden de distribución de los cuerpos en funciones correspondientes a su 'naturaleza' y en lugares correspondientes a sus funciones...» (Ranciere,1996:128).
} 
de dos manos, que en lugar de agotar las energías utópicas, se apoye en el experimentalismo presente en los ensayos y en las políticas instituyentes. Para el caso de la administración de la locura debemos recuperar el modelo «bottom up», es decir, un modelo de trabajo que se soporta en proponer la idea de administración pública como administración de la crítica, en donde lo que sostiene un modelo mutante de gestión, es la tensión dialéctica de abajo hacia arriba, y el juego estratégico de afuera hacia adentro.

Prácticas de gobierno (con ' $g$ ' minúscula como señalaría Carlos Matus) que hoy debemos reconocer y defender para una serie de casos en la Argentina ${ }^{3}$, no para sacralizarlas, sino sobre todo para ampliar el discurso de lo posible en el terreno de las prácticas de salud mental. Darle visibilidad opera un doble objetivo: primero, un paso para su defensa, y segundo ( $y$ no por eso menos importante), un paso también para el contralor público de la misma y su no cristalización y serialización.

Si al principio hablábamos de rehabilitar las reformas, debemos también rehabilitar los contenidos instituyentes de nuestra historia en una genealogía que haga justicia so pena de quedar anclados en los contenidos conservadores de nuestra historia, como la Coordinadora de Trabajadores de la Salud Mental o los movimientos de Plataforma y Documento, no para ritualizarlos sino para promover su actualización para la construcción de un nuevo disenso, en el que la propia herramienta se complejice e incluya nuevos actores, como los usuarios, sus familiares, artistas, enfermeros, trabajadores sociales, etc.

La materia prima de esta agenda de reformas puede encontrarse en una serie de grietas y fisuras en el muro levantado por el consenso neoliberal, permitiendo la construcción de nuevas afiliaciones, experimentales y provisorias, conjugadas con nuevos espacios públicos de asistencia, siendo posible nombrar entre las más relevantes de las distintas inscripciones trabajadas a lo largo del texto las siguientes vías:

La vía de las ciudadanía tras-locada. Consideramos que es una estrategia central a los efectos de incluir la vigencia de la desrazón en el territorio de la ciudad, insertar las prácticas de asistencia en salud mental dentro del campo y la conquista de la reinvención permanente de la ciudadanía democrática, implicando la construcción de dispositivos de lucha por el ejercicio de los tradicionales derechos civiles, políticos y sociales por parte de la minoría portadora de la desrazón. Estos dispositivos suponen una lucha específica por el reconocimiento de la ciudadanía «especial» a ser re-inventada, tanto por el reconocimiento de los usuarios y familiares en la producción de los servicios, como por una ciudadanía que integre la diferencia, el conflicto y la desrazón como propias, por lo tanto una ciudadanía pos pero necesariamente moderna, no consiguiendo ser encontrada esta ciudadanía en las concepciones modernas racionalistas y universalistas asociadas al individuo racional, libre y autónomo. Esta ciudadanía -la tradicional moderna- se constituye más en una barrera que en un avance a la ciudadanía del loco (Mouffe,1996:98), siendo fundamental para la revolución democrática, constituye hoy, sin embargo, un obstáculo para su extensión. No olvidemos cómo los teorizadores de la historia de la locura y las feministas han registrado; que el dominio de lo público, fue basado en el

\footnotetext{
${ }^{3}$ Para un adecuado estudio de los «casos» de reforma en la Argentina refiero al libro de mi autoría Reformas y contra-reformas. Políticas de Salud Mental en Argentina. UNR Editora. Rosario. 2003
} 
rechazo a la participación de las mujeres, del loco y de otras minorías ${ }^{4}$ en el mismo. La construcción actualizada de una (des-) razón emancipatoria se conjuga con la invención de una interpretación democrática radical de los principios liberal-democráticos «...tal interpretación presupone que estos principios sean entendidos de forma que se tornen en consideración las diferentes relaciones sociales y posiciones del sujeto en que son relevantes: género, clase, raza, etnia, orientación sexual, (des-razón), etc...» (Mouffe, 1996: 97)

Este «nuevo público» elige como opción prioritaria la des-psiquiatrización de la asistencia de la minoría pasible de la asistencia «psiquiátrica», entendida como aquella más vulnerable a ocupar una cama en el circuito de hospitalización psiquiátrica clásica. Esto implica poner entre paréntesis la opción sanitarista que tiende a inducir difusamente por toda la sociedad demandas de cuidados en salud mental y se desresponsabiliza en la práctica de romper el circuito de internación. La responsabilidad del derecho a la asistencia priorizará en esta otra lógica las cambiantes necesidades de autonomía de esta «vieja» minoría y hará uso de la caja de herramientas del análisis institucional y de la genealogía, buscando argumentos para cuestionar relaciones cotidianas de dominio a fin de que los principios de libertad e igualdad puedan aplicarse en lo cotidiano de las viejas y nuevas instituciones.

La vía del welfare artesanal. Para sostener la economía política de la desmanicomialización es nodal recuperar las intuiciones centrales del concepto acuñado en Trieste «el welfare artesanal», de hecho frente al híbrido estatal-privatista multiplicador de la exclusión y la desafiliación, debemos promover en la práctica la reconstrucción creativa y democrática del Welfare. Frente al circuito neoasistencial, clínica privada-tribunales-dispensariosmanicomios-hospitales de urgencia, debe ganarse a la seguridad social para un nuevo circuito de asistencia y un nuevo híbrido: densificación de la asistencia de los servicios públicos locales-organizaciones de usuarios y de trabajadores de la salud mental. Si más acá del Estado y más allá del Mercado, las reformas deben encontrar líneas de administración públicas, la cuestión central es traer para el sector de salud mental los indicativos de reproducción social del movimiento por la defensa del derecho a la ciudad. Siendo básico encontrar mecanismos de democratización de los financiamientos públicos de salud, incorporando criterios ético-estéticos para el apoyo a la construccióndeconstrucción de nuevos servicios de asistencia ${ }^{5}$, diferentes de las razones (y

\footnotetext{
${ }^{4}$ La construcción de este largo disenso, que convalida como motor de los cambios la ciudadanía de los grupos oprimidos, no establece como dadas las afiliaciones entre los mismos, sino como un proceso sumamente conflictivo en el que deben estar presentes altos niveles de diálogo. Considero interesante colocar un ejemplo para fundamentar este llamado de atención, poner en consideración los términos de la relación entre las personas que padecen sufrimiento psíquico y las feministas: desde hace bastante tiempo las feministas vienen interpelando las políticas de desinstitucionalización por sus efectos de descarga del problema en la familia y especialmente en la mujer en la obligación de los cuidados, una política de desmanicomialización deberá tomar altamente en cuenta este reclamo para la democratización de la asistencia y para no producir nuevas «cargas» sociales y reificar (in-voluntariamente) la condición subordinada femenina.

${ }^{5}$ Si no se avanza en la construcción de servicios alternativos al manicomio, como Centros Comunitarios de Salud Mental que funcionen las 24 horas, Cooperativas de Trabajo y Centros Culturales y de Acompañamiento Diario co-gestionados con los usuarios, se perderán «oportunidades históricas» y la mayor parte de los recursos continuarán presupuestando los manicomios y las administraciones «progresistas» continuarán salvando la institución totalitaria y reduciendo las políticas de externación a un mix de soluciones familiaristas con aportes comunitarios sumamente precarizados.
} 
sus externalidades irracionales) económicas del circuito privado, demostrando pragmáticamente mayor eficacia en términos de ciudadanía e integración social y de una cogestión mejor sucedida: servicios públicos más organizaciones de usuarios y defensores de los derechos humanos.

La construcción de un frente político (no sólo enunciado desde los «profesionales psi» sino textado desde la cotidianeidad de trabajadores, usuarios, familiares, ciudadanos, etc.) que recupere la experiencia de otros movimientos sociales (mujeres, desocupados, discapacitados) en las políticas de advocacy y en el control público de la gestión estatal, aparece como una de las llaves para llevar adelante la construcción de nuevos servicios públicos, especificando la posibilidad de presionar por una reformulación conjunta de principios y procedimientos, líneas de trabajo que apuesten en avanzar de la tutela al contrato, multiplicando en sus «agentes» (lo contrario de pacientes) capacidades sociales, recursos de singularización, participación en derechos e intercambios sociales. Construcción de un frente político que debe afrontar el trabajo del pasaje de los derechos al Derecho en el caso de la locura, que no puede ser visto solamente como una simple traducción de demandas «progresistas» en un lenguaje jurídico formal sino que centralmente debe consistir en la elaboración, tanto política como institucional y presupuestaria, de los nuevos derechos para que sean efectivamente asumidos y respetados por un sistema como el político y el jurídico, más preocupados siempre por el control de los riesgos que por las políticas activas de construcción de ciudadanía.

Pero también la construcción de un movimiento social y de un frente discursivo de «una sociedad sin manicomios» debe estar atento a realizarse una serie de cuestionamientos sobre cómo se procesan los debates sobre la realidad de los manicomios y la situación de los pacientes internados. Por ejemplo, en el tratamiento que hace la prensa, cuáles son las ventajas que los «locos» realmente existentes obtienen de las coberturas mediáticas amarillas o de las crónicas negras de los manicomios y/o inclusive del «elogio de la locura» que reduce todo a una política de las buenas intenciones.

Esto no significa rechazar el tratamiento público del problema, ya que se hace urgente ganar un espacio considerable en las agendas políticas dirigidas a la disminución de la discriminación y el racismo institucionalizado. Sin embargo, debemos poner en tela de juicio el papel que le cabe al nuevo periodismo y a los medios de comunicación «solidarios», que bajo el velo de la denuncia, generan una simplificación radical de la lectura del problema de los manicomios en una mixtura entre el sensacionalismo de lo inaceptable y el unanimismo de lo deseable. 


\section{Políticas de la locura, redistribución y reconocimiento (o recordando a Marx, a cada uno según sus necesidades, a cada uno según sus capacidades)}

Hablar de políticas de salud mental ${ }^{6}$, de representación dentro del espacio público de la problemática de la locura, nos obliga a reflexionar sobre cuáles son los criterios consistentes de justicia social en esto de llevar adelante la vigencia de los derechos humanos para el caso de la salud mental.

En este sentido quiero retomar una autora, N. Fraser (1998) quien, desde la sociología política, revisa las principales ideas de justicia social presentes en la tradición anglosajona y realiza una proposición bastante saludable, sobre todo para el caso que nos interpela. La autora reconoce dos paradigmas principales en esto de producir justicia social: el paradigma más tradicional de la redistribución, que reclama un reparto más justo de recursos y bienes en el sentido de la igualdad; y el paradigma del reconocimiento que ejerce un tipo de reclamo en el sentido de la diferencia, es decir, un mundo que acepte las diferencias en el que la asimilación a las normas culturales dominantes de la mayoría ya no sea el precio a pagar por un respeto equitativo, línea reconocida más clásicamente como las políticas de identidad: minorías étnicas, sexuales, diferencias de género, de desrazón, etc.

Paralelamente, la autora observa que en la actualidad la primera está notoriamente a la defensiva debido al consenso neoliberal y también se evidencia una serie de falsas antinomias entre ambas. Dentro de marcos más ortodoxos, las políticas de la diferencia son vistas como falsa conciencia y dentro de las políticas de la identidad más relativistas la cuestión del materialismo está fuera de moda. Y de forma menos cuidadosa se escucharía: «es la economía estúpido; no, es la política estúpido».

La autora promueve y previene sobre la importancia de recuperar los aspectos emancipatorios de ambas matrices en un único marco comprehensivo, sin reducir la una a la otra en la producción de una concepción bivalente de justicia. Hagamos ahora un ejercicio que nos permita observar los remedios que orienta cada una en el terreno de la justicia social, sus colectivos sociológicos de preferencia en cuanto a las reivindicaciones y sus matices en relación a los factores que generan la desigualdad y la discriminación cultural.

Las políticas de la redistribución apuntan, como principal remedio para superar condiciones de explotación y marginación, a la redistribución social y económica propuesta históricamente por el socialismo, que se constituirían en la mejor manera de solucionar los problemas de desigualdad y las injusticias de género, raciales, étnicas, etc. La colectividad (el grupo beneficiario) de

\footnotetext{
${ }^{6}$ A lo largo del trabajo entendemos por conceptuación en políticas de Salud Mental, no las más cercanas a las clásicas definiciones de la OMS, sino el conjunto de prácticas políticas y técnicas vinculadas al campo de saberes que transversalizan al campo de saberes de la psiquiatría, el psicoanálisis y el sanitarismo. En la salud mental lo que está en cuestión son los procesos y estrategias de subjetivación, así como los destinos sociales de los sujetos que padecen sufrimiento psíquico. Desde este punto de vista se torna evidente que toda discusión técnica de las formas de tratar con la locura es al mismo tiempo una reflexión política sobre las condiciones de ciudadanía de los usuarios, la institución psiquiátrica en su autonomía relativa, es decir en sus conceptos, prácticas, normas, establecimientos asistenciales, o sea, el modo instituido de tratar a los locos es extremadamente revelador de los impasses y contradicciones que atraviesan las políticas sociales.
} 
preferencia de este paradigma, recordemos, es la clase que vive del trabajo y también, para ser justos con la tradición marxista, distintas infra-clases, es decir aquellos excluidos del trabajo asalariado y considerados por los sectores dominantes como los sectores indignos de ser explotados. El paradigma de la redistribución claramente se confronta con aquellos diferenciales injustos que deben ser abolidos. El problema de base estaría en la economía política y en la injusta distribución socio-económica.

Las políticas del reconocimiento apuntan, como principal remedio para dar respuesta a la discriminación social, a una fuerte política de inversión del estigma, de identidades injustamente devaluadas (el negro, la mujer, el loco, etc.) y aboga por un cambio cultural y simbólico que promueva la revalorización ascendente de identidades menoscabadas. El paradigma del reconocimiento reconoce como grupo beneficiario a aquellos sin estatuto, es decir el tipo más weberiano, cuyo ejemplo clásico es el grupo étnico considerado de baja condición, cuyos miembros son señalados como diferentes y menos dignos por los patrones de interpretación y valoración dominantes. En cuanto a las diferencias, el paradigma del reconocimiento las considera, por un lado, valoraciones culturales que deberían celebrarse y, por otro, como oposiciones jerárquicas construidas discursivamente que deberían deconstruirse. El problema de base estaría en el orden sociocultural y estamental.

Hecho este ejercicio, la autora realiza una crítica de ambas escuelas, una crítica de esta justicia interruptus, y en base a un efecto de demostración en relación a una serie de ejemplos, observa que cada vez más cualquier colectivo discriminado registra formas tanto de mala distribución socioeconómica como de no reconocimiento social y cultural y que una no es efecto indirecto de la otra, sino que ambas son primarias y co-originarias. El ejercicio de la autora es arriesgado y demuestra la hibridez para el caso de las políticas de género e inclusive de las políticas de clase. Refiere, por ejemplo, la importancia que tienen en términos de hegemonía, las ideologías de la «cultura de la pobreza», que en sus estrategias de criminalización e infantilización de la misma, ayudan a naturalizar la marginación y la exclusión socio-económica.

Para el caso que nos convoca, la locura, observamos la importancia tanto de trabajar en el terreno de la redistribución como en el del reconocimiento ${ }^{7}$, como así también observar que los locos con los que trabajamos pertenecen a lo que la autora conceptualiza como colectivos bivalentes. Por ejemplo, en el Welfare corporativo argentino las políticas de redistribución hacia la problemática de la locura, fueron en su momento funcionales al manicomio, y por lo tanto al mantenimiento de la discriminación cultural del loco.

Si no entendemos esto, estamos errando, el problema no es sólo más presupuesto, sino cuáles son los usos de justicia social de ese presupuesto. Las colectividades, por ejemplo, que son asistidas en los manicomios,

\footnotetext{
${ }^{7}$ Esta política de inversión del estigma debe recordar la lección del maestro Irving Goffman, es decir, que la persona que sufre un estigma se debate entre su negación del estigma y su deseo de superar su condición, pues se siente plenamente humano pero paralelamente portador de una humanidad «anormal» en relación con los estatutos científicos a los que la sociedad adhiere. La tensión se convierte así en una fuente de ambigüedad en el terreno simbólico de la acción política: la movilización política debe responder a la doble exigencia de un reconocimiento de derechos especiales en virtud de su condición «diferente» y paralelamente a la necesidad de despegarse de la etiqueta (o el diagnóstico) de esta identidad estigmatizada y de su derecho a ser reconocido como un «igual».
} 
participan tanto de problemas de clase (infraclase: los casos sociales) como de estatuto (segregación cultural: normal-patológico), como ya lo observaba hace 30 años Basaglia en la mayoría desviante.

Por otro lado, debemos salirnos de la explicación reduccionista y económico-legalista que supone que una justa distribución de recursos y derechos formales es suficiente para eliminar las distintas formas de discriminación negativa. Como se diría en otros campos: condición necesaria pero no suficiente, mayor presupuesto para el caso de la salud mental no asegura per se cambios en las patrones culturales y asistenciales de quienes tienen el mandato social de «administrar» el problema de la locura.

Considero que esta concepción bivalente de la justicia social -y entiendan bien jugando con la retórica los psicoanalistas y no del bien o de la vida buena, o sea una política de justicia que implique «términos justos de interacción bajo la condición de un pluralismo de valores» y no «medicalización» de la felicidad de un Estado Pastoral- permite un juego de dos velocidades que enfrente a la fábrica de consenso de la psiquiatría contemporánea reconocida como salud mental y para nosotros servicios «privados» de salud mental ${ }^{8}$.

\section{Reflexiones finales}

Como conclusión, no quiero escapar de un planteo de la autora que me parece central para el plano de los cambios en la cultura de los servicios asistenciales de salud mental y que para nuestro caso ya criticaba Foucault en su relectura del «elogio» de la locura de Erasmo de Rotterdam. La autora se pregunta «qué» diferencia y en este sentido, frente a los esencialismos de la diferencia, la autora los rechaza de plano y promueve un enfoque «pragmático» de las diferencias, es decir, el reconocimiento de las diferencias que la justicia requiere en un caso depende de las formas de la discriminación a ser enmendadas. O sea, frente a los universalismos abstractos promueve universales de la diferencia construidos socio-históricamente «...todo depende de qué es, precisamente, lo que las personas que hoy son segregadas necesitan para poder participar como pares en la vida social, ya que no hay razón para suponer que todos ellos necesitan lo mismo en cualquier contexto. En algunos casos, pueden necesitar ser liberados de rasgos distintivos que se les adjudican. En otros casos pueden necesitar que se tomen en cualidades distintivas, no suficientemente reconocidas hasta ahora, mientras que en otros, incluso, pueden necesitar desplazar la atención hacia los grupos dominantes y aventajados, revelando esos rasgos distintivos que habían sido falsamente exhibidos como universalidad. Alternativamente pueden necesitar deconstruir los términos mismos en los cuales se formulan habitualmente las diferencias atribuidas. Finalmente pueden necesitar todas o varias de estas cosas combinadas con la redistribución...» (Fraser, 1998:33)

\footnotetext{
${ }^{8}$ Fábrica de consenso de la psiquiatría contemporánea, en que pacíficamente directores de las instituciones públicas de salud mental, pueden por otro lado participar sin «contradicciones» cómo accionistas de clínicas privadas psiquiátricas.
} 
Me parecen centrales estos planteos para guiar tanto las estrategias políticas como asistenciales en el caso de la salud mental ${ }^{9}$, el elogio de la diferencia puede llevarnos a una estética cool de la locura, mostrándonos como nuevos filántropos y/o alienistas, cayendo en la versión más protegida de la asistencia y que tranquilamente puede convivir con la «demonización» de la diferencia, en que locura y peligrosidad van de la mano en las páginas policiales y no sólo en ellas.

Finalizando, ahora sí, me viene a la memoria la conclusión de Castel respecto de los límites y el voluntarismo de los movimientos psi contra el orden psiquiátrico, y considero que la conclusión de esta cita es importante a la hora de reflexionar sobre cómo participar de este movimiento social por el adiós a los manicomios:

«...A falta de una revisión de esta situación, el desprecio de las abstracciones de la vieja política induce una forma sutil de psicologismo por la cual el sujeto se ha convertido en el último objetivo legítimo de un proceso de transformación completamente banalizado que se jacta todavía, no se sabe bien por qué, de las virtudes del progresismo. La desconfianza respecto de los poderes centrales, de las organizaciones estructuradas, desemboca en la apología de la sociabilidad convivencial en la que los problemas de la vida cotidiana se autogestionen en un marco asociacionista que hace de los militantes reconvertidos los herederos de las viejas damas de beneficencia...» (Castel, 1984:14).

\section{Bibliografía}

AMARANTE, P. O planejamento na deconstrução do aparelho manicomial. Rio de Janeiro, Relumé Dumará, 1992.

BASAGLIA, F. «Consideração sobre uma experiência comunitária» en: AMARANTE, P. Psiquiatria social e Reforma Psiquiátrica. Rio de Janeiro, FIOCRUZ, 1994.

BIRMAN, J. «A cidadania traslocada» en: BEZERRA, B. Psiquiatria sem hospício: Contribuições ao estudo da reforma psiquiátrica. Rio de Janeiro, Relume Dumará, 1992.

BOSCH, G. «El Estado pavoroso de la locura en la Argentina» en Revista Medicina Argentina, N X, 1931.

\footnotetext{
9 «...la transformación más decisiva intervenida después de una decena de años en el sector de la medicina mental, fue sin duda un retroceso de los que se podría llamar de racismo antiloco, una de las formas más profundamente inscriptas de negación de la diferencia. No habría ahí, sino su contribución a depositar en el crédito de la antipsiquiatría a esa mudanza, aquellos que de ella participaron o por ella fueron asimilados no tendrían que lamentarse de haber destinado algún esfuerzo. Es en la medida que ese tipo de adquisición es siempre frágil, provisoria, amenazada, donde en fin todos los racismos subterráneos se dan la mano en la gran comunión de los excluyentes, valdrá todavía la pena consagrar esfuerzos en el futuro...» (Castel,1984:29)
} 
CASTEL, R. El Orden Psiquiátrico, la edad de oro del alienismo. Madrid, Ediciones La Piqueta, 1978.

CASTEL, R. La gestión de los riesgos. Barcelona, Anagrama, 1984.

FRAZER, N. «La justicia social en la era de las políticas de identidad» en Revista de Sociología Apuntes de Investigación, № 2/3. Buenos Aires, Edición Fundación del Sur,1998.

MOUFFE, C. O regresso do político. Lisboa, Gradita, 1996.

RANCIERE, J. El Desacuerdo. Buenos Aires, Nueva Visión, 1996. 